\title{
Outcomes of Cochlear Implantation in Post-lingually Deaf Patients
}

Saeedi $\mathbf{M}^{1,3}$

Amirsalari $\mathrm{S}^{2}$

Dadgar $\mathrm{S}^{3}$

Khosravi $\mathrm{MH}^{4}$

\begin{abstract}
Introduction: Cochlear implantation has been mentioned as the most effective therapeutic intervention in deaf patients and especially those with post-lingual deafness. We aimed to assess hearing improvement of post-lingually deaf patients after cochlear implantation.
\end{abstract}

Materials and methods: in this cross-sectional study all the post-lingually deaf patients who had undergone cochlear implantation (Cl) surgery between December 2010 and February 2016 were assessed. Patients were recalled and after explaining the study process and signing an informed consent form, an audiometry was done by a single audiologist. In addition, demographic information, cause of hearing loss, age of onset, history of hearing aid use and surgical complications were recorded in a pre-designed checklist.

Results: Twenty-nine male and 21 female with a mean age of $22.52 \pm 19.45$ years underwent analysis. Most of patients (80\%) had progressed condition since childhood. Sudden sensorineural hearing loss (SSNHL) was the most prevalent (10\%) known etiology of deafness followed by meningitis (6\%), Trauma (2\%) and ototoxic drugs $(2 \%)$ in the remaining $20 \%$ of the patients. Patients had the highest mean $(37.1 \pm 12.61 \mathrm{~dB})$ in $4000 \mathrm{~Hz}$ frequency and the lowest mean of hearing threshold level $(32.6 \pm 8.37 \mathrm{~dB})$ was for 1000 $\mathrm{Hz}$ frequency. Mean hearing threshold level was significantly lower in patients with lower ages of cochlear implantation $(p=0.435)$. Patients with higher ages of deafness onset showed lower degree of hearing improvement $(p=0.462)$.

Conclusion: The results of our study suggest that cochlear implantation significantly improves hearing function of post-lingual patients and can be considered as a certain cure for these patients in Iran.

Keywords: Tinnitus, Cochlear implantation; Post-lingually deaf patients; Complications; Efficacy; Outcome

\footnotetext{
1 Department of Otorhinolaryngology, Head and Neck Surgery, Faculty of Medicine, Baqiyatallah University of Medical Sciences, Tehran, Iran 2 Department of Cochlear Implantation Research Center, Baqiyatallah University of Medical Sciences, Tehran, Iran

3 Department of Otorhinolaryngology Research, Universal Scientific Education and Research Network (USERN), Tehran, Iran

4 Department of Otolaryngology, Faculty of Medicine, Tehran University of Medical Sciences, Tehran, Iran
} 


\section{INTRODUCTION}

Deafness, with a prevalence of 2 to 4 per 1000 people in United States, is one of the most important disorders which has remarkable impacts on daily activities of patients; so that even those with low levels of hearing disorders are involved with these problems. Severe impairment of daily life, having higher risks of physical damage and negative psychological consequences are among deafnessderived problems ${ }^{1,2}$. Post-lingual deafness is defined as hearing loss which develops after the acquisition of speech and language, usually after the age of six; however it may begin to develop in lower ages ${ }^{3}$. There are a variety of reasons known to cause deafness. In the United States, $56 \%$ of cases are of unknown cause and genetic disorders are responsible for $23 \%$ of deafness in children. Acquired causes like pre-, peri- and postnatal events as well as other reasons such as tumors, congenital malformations and cysts cover $20 \%$ and $1.2 \%$ of the cases, respectively ${ }^{4}$. Also previous studies have indicated that incidence of deafness is inversely related with socioeconomic situation of families ${ }^{5}$. In the last decade, various methods have been proposed for improving hearing and communication in deaf patients and those with mild hearing impairment. Using hearing aids and learning skills such as lip reading and sign language as well as cochlear implantation are among these methods ${ }^{6}$. In the last years, cochlear implantation has been mentioned as the most effective therapeutic intervention in deaf patients and especially those with post-lingual deafness 7. Cochlear implant not only improves hearing perception, speech and communication ability, but also has beneficial effects on non-audiological predictors such as self-esteem and coping strategies of patients ${ }^{8}$. Cochlear implantation has also significantly improved quality of life of post-lingual patients ${ }^{9}$. Previous studies have assessed effective factors of hearing condition and speech recognition of deaf people after cochlear implantation; however in general post-lingual patients have been less studied in comparison with pre-lingual cases. Regarding that no similar studies have been yet conducted in Iran, we aimed to assess hearing improvement of post-lingually deaf patients after cochlear implantation.

\section{METHODS}

This Cross-sectional study was conducted between December 2010 and February 2016 in Baqiyatallah university hospital, Tehran, Iran. This study was registered at ethics committee of Baqiyatallah University of Medical Sciences. All the post-lingually deaf patients who had undergone cochlear implantation $(\mathrm{Cl})$ surgery in this time period were assessed for eligibility. Patients more than 6 years of age for whom it was not possible to assess the hearing and speech test before $\mathrm{Cl}$ as well as those with "severe to profound" result in auditory brainstem response (ABR) were included in the study. Patients with neurological damages or other associated disabilities as well as those not willing to participate were excluded from the study. Patients were recalled and after explaining the study process and signing an informed consent form, an audiometry was done by a single audiologist. In addition, demographic information, cause of hearing loss, age of onset, history of hearing aid use and surgical complications were recorded in a pre-designed checklist. Data were analyzed using SPSS software version 21 (SPSS Inc., Chicago, IL) for Microsoft Windows. Nonnormal distributed variables (approved by 1-sample Kolmogorov-Smirnov test) were compared using Mann Whitney $\mathrm{U}$ test between the groups. Pearson's correlation coefficient was used for evaluating relations between quantitative variables. The chi square test was used to compare categorical variables in the 2 groups. Mean and standard deviation (SD) were used for describing categorical variables.

\section{RESULTS}

Eventually 50 patients (29 male and 21 female) with a mean age of $22.52 \pm 19.45$ years underwent analysis. Half of the patients had undergone cochlear implantation in right ear and the remaining half in the left with a mean duration of $2.18 \pm 1.09$ years. Age of hearing loss onset had a mean of $15.90 \pm 20.45$ years among study individuals. Most $(84 \%)$ of the patients had no positive history of hearing loss or deafness among their families. Out of study individuals $45(90 \%)$ patients had a positive history of hearing aid use for a mean duration of $2.62 \pm 2.86$ years. Table 1 summarizes the distribution of associated diseases in study individuals. Thirty-five (70\%)

Table 1: Distribution of associated conditions among study individuals.

$\begin{array}{cc}\text { Condition } & \mathrm{N}(\%) \\ \text { Seizure } & 3(6 \%) \\ \text { Vision problems } & 1(2 \%) \\ \text { Hypertension } & 2(4 \%) \\ \text { Cleft lip } & 1(2 \%) \\ \text { Diabetes Mellitus } & 1(2 \%) \\ \text { Delayed speech } & 1(2 \%) \\ \text { Proteinuria } & 1(2 \%) \\ \text { ADHD } & 2(4 \%) \\ \text { Acoustic tumor } & 1(2 \%) \\ \text { Concurrent Vision problems and Acoustic tumor } & 1(2 \%) \\ \text { Concurrent HTN and DM } & 1(2 \%)\end{array}$


Table 1: Frequency of different hearing threshold levels in patients after cochlear implantation.

$\begin{array}{cc}\text { Mean hearing threshold level (dB) } & \mathrm{N}(\%) \\ 20-30 & 18(36 \%) \\ 30.01-40 & 21(42 \%) \\ 40.01-50 & 4(8 \%) \\ 50.01-60 & 5(10 \%) \\ 60.01-70 & 2(4 \%)\end{array}$

Table 3: Mean hearing threshold level of different evaluated frequencies in study individuals after cochlear implantation

$\begin{array}{cccc}\text { Frequency } & \begin{array}{c}\text { Minimum hearing threshold } \\ (\mathbf{d B})\end{array} & \begin{array}{c}\text { Maximum hearing threshold } \\ (\mathbf{d B})\end{array} & \begin{array}{c}\text { Mean } \\ (\mathbf{d B})\end{array} \\ \mathbf{2 5 0 ~ H z} & 20 & 55 & 32.6 \pm 9.26 \\ \mathbf{5 0 0 ~ H z} & 20 & 60 & 33.8 \pm 8.85 \\ \mathbf{1 0 0 0 ~ H z} & 20 & 50 & 32.6 \pm 8.37 \\ \mathbf{2 0 0 0 ~ H z} & 20 & 80 & 34.3 \pm 10.59 \\ \mathbf{4 0 0 0 ~ H z} & 20 & 100 & 37.1 \pm 12.61 \\ \mathbf{8 0 0 0 ~ H z} & 10 & 100 & 36.3 \pm 15.21 \\ \text { Total Mean } & 18.3 & 74.1 & 34.4 \pm 9.68\end{array}$

of patients had no underlying or associated conditions. A majority of patients $(80 \%)$ reported that their condition has progressed since childhood. Sudden sensorineural hearing loss (SSNHL) was the most prevalent (10\%) known etiology of deafness followed by meningitis (6\%), Trauma (2\%) and ototoxic drugs (2\%) in the remaining $20 \%$ of the patients. Unhealed wound was the most common (8\%) surgical complication followed by surgery site infection (4\%); while $44(88 \%)$ patients had no surgical complications. It frequency of different hearing threshold levels in patients after cochlear implantation. Most part $(42 \%)$ of patients had a mean hearing threshold level between 30.01 and $40 \mathrm{~dB}$. Only 2(4\%) patients had a mean hearing threshold level between 60.01 and $70 \mathrm{~dB}$. Mean hearing threshold level of different evaluated frequencies in study individuals has been summarized in Table 3 . Patients had the highest mean $(37.1 \pm 12.61 \mathrm{~dB})$ in 4000 $\mathrm{Hz}$ frequency and the lowest mean of hearing threshold level $(32.6 \pm 8.37 \mathrm{~dB})$ was for $1000 \mathrm{~Hz}$ frequency. Mean hearing threshold level was significantly lower in patients with lower ages of cochlear implantation $(p=0.435)$. There was no significant difference between two genders for hearing improvement after cochlear implantation $(p=0.927)$. Patients with higher ages of deafness onset showed lower degree of hearing improvement $(p=0.462)$. There was no correlation between positive family history of deafness and improvement of hearing after cochlear implantation $(p=0.864)$. Patients with underlying disease and associated conditions showed a significantly lower improvement of hearing after cochlear implantation $(p<0.01)$. Patients with history of hearing aid use had significantly higher hearing thresholds after cochlear implantation $(p<0.001)$. However; patients with longer duration of hearing aid use showed higher improvements in hearing $(p=0.042)$. There was no significant association between implanted ear (left or right) and hearing improvement $(p=0.610)$. Also there was no significant correlation between past years of cochlear implantation and improvement of hearing threshold $(p=0.24)$. Mean hearing threshold level was significantly lower in patients with no surgical complications in comparison with those who had unhealed wound or infection $(p<0.001)$.

\section{DISCUSSION}

Estimates have shown that 2 to 4 people out of 1000 are functionally deaf in United States; however more than half of these population get deaf in older ages and less than one in thousand are deaf before eighteen years of age. In brief, 37 to 140 people out of 1000 have mild to severe hearing problems in United States which a great proportion of them are those above 64 years old 2 . Although in a recently published study researchers have reported that prevalence of hearing loss in United States has decreased from $16 \%$ in 2004 to $14 \%$ in $2012^{10}$. They have mentioned less manufacturing jobs, less smoking, more using of hearing protectors and progresses in health care as the suggested causes of the decline. Unfortunately there is no accurate statistics available for prevalence and causes of hearing loss in Iran. In a single study, Hajloo et al ${ }^{11}$. have reported that 7.1 per thousand people suffer from hearing impairment and 4.3 per thousand are deaf in western Iran ${ }^{11}$. Comparing the results of hearing and speech tests after cochlear implantation $(\mathrm{Cl})$ in prelingual patients with and without inner ear problems, Akhavanfar et al. concluded that there are no significant relation between gender and improvement in hearing function after $\mathrm{Cl}^{12}$. They have reported an improvement in speech tests after $\mathrm{Cl}$. A few studies have been conducted to assess the causes of post-lingual deafness. In these studies developing hearing loss since childhood, trauma and ototoxic drugs have been counted among etiologies of post-lingual deafness ${ }^{13,14}$. In Ahmad et al. study, postlingual deaf children showed a significant improvement in open-set speech perception tests 6 months after $\mathrm{Cl}$; while it was 24 months for pre-lingual deaf ones ${ }^{15}$. In this study, it was determined that children with lingual skills prior to $\mathrm{Cl}$ showed an earlier improvement in a two-year follow up and they are better candidates for cochlear implantation. 
In another similar study, Tanamati et al. evaluated speech perception in post-lingual deaf children after $\mathrm{Cl}$. They have concluded that $\mathrm{Cl}$ is a safe and reliable method for treatment of these patients and induces a significant improvement in speech recognition in a ten-year follow up ${ }^{16}$. Cochlear implantation was also reported to be beneficial for improving hearing function in post-lingual deaf patients and especially those with bilateral profound deafness ${ }^{17}$. Hamzavi et al ${ }^{17}$. mentioned that hearing function was improved following $\mathrm{Cl}$ after 12 months of surgery in their adult post-lingual patients 18. They also concluded that age of implantation, age of onset and duration of deafness as well as depth of implantation are among important predictive factors of hearing condition one year after $\mathrm{Cl}$. In another similar study, post-lingual patients were evaluated in both quiet and noise situations after cochlear implantation. It was determined that $\mathrm{Cl}$ significantly improves speech function of patients and that factors like duration of deafness, concurrent use of hearing aids, pure tone audiometry (PTA) of healthier ear, type of applied implant and percentage of active electrodes are significantly correlated with speech function. This is in accordance with Kraaijenga et $\mathrm{al}^{18}$. study however age of $\mathrm{Cl}$ was not an determining factor of $\mathrm{Cl}$ function in their study ${ }^{19}$. In a review on 533 cases of severe to profound deafness (93\% pre-lingual and $7 \%$ post-lingual), who had 1 to 17 years of age at the time of $\mathrm{Cl}$, satisfaction for hearing improvement was $94.6 \%$ in pre-lingual and $70 \%$ in post-lingual patients ${ }^{20}$. Most of the previous studies have focused on the effects of $\mathrm{Cl}$ on speech perception. In addition to above mentioned effects, $\mathrm{Cl}$ can also improve quality of life of post-lingual deaf patients ${ }^{21}$. Lassaletta et al. used Glasgow Benefit Inventory for evaluating quality of life and communication skills in 30 postlingual deaf patients who had underwent $\mathrm{Cl}$. This study showed positive effects of $\mathrm{Cl}$ on using telephone and selfconfidence as well as significant improvement of quality of life so that $96 \%$ of patients recommended $\mathrm{Cl}$ to their friends. Bittencourt et al ${ }^{21}$. evaluated benefits of cochlear implants versus conventional hearing aids in a review article. They found that $\mathrm{Cl}$ is a more effective method with better outcomes in comparison to hearing aids according to speech perception testing, Minimal Auditory Capabilities (MAC), Hochmair-Schulz-Moser (HSM), Hearing in Noise Test (HINT) and other common tests. Cochlear implantation may guarantee voice perception and speech recognition ability with near normal hearing; however there is a difference between implants in terms of function in speech differentiation and recognition tests. de Brito et al ${ }^{22}$, in a retrospective study, found that post-lingual patients who had underwent $\mathrm{Cl}$ because of meningitis had weaker results in speech recognition tests in comparison with those with other etiologies of deafness. This difference was evident in complex hearing actions such as harmony singing ${ }^{23}$. A variety of studies have reported that outcomes of $\mathrm{Cl}$ is postponed in post-lingual deaf patients with long-term problem and sometimes have had unsatisfactory results in comparison with those who have gotten deaf more recently; however history of long- term deafness is not a contraindication for $\mathrm{Cl}$. It has been shown that patients with long-term problems may have satisfactory functional results although with a lower speed and also using bilateral cochlear implantation accelerates final outcome ${ }^{24}$. The present study has some limitations. Patients with traumatic or ototoxic etiologies of deafness were in the minority. Also the present study has a low geographic span because of problems in transportation for patients; so a few study individuals were from other parts of the country. Evaluating post-lingual patients in Iran for the first time and a relatively large sample size are among strengths of the present study.

\section{CONCLUSION}

In conclusion, the results of our study suggest that cochlear implantation significantly improves hearing function of post-lingual patients and can be considered as a certain cure for these patients in Iran. Also we realized that etiology and age of onset of deafness, non-use or duration of hearing aid use and incidence of post-surgical complications are effective factors on hearing threshold levels after cochlear implantation.

\section{CONFLICT OF INTEREST}

There are no conflicts of interest in terms of the present study.

\section{REFERENCE}

1. Pourabdian S. The prevalence and correlates of hearing loss in drivers in Isfahan, Iran. Archives of Iranian medicine. 2009;12(2):128-34.

2. Schley S, Walter GG, Weathers RR, Hemmeter J, Hennessey JC, Burkhauser RV. Effect of postsecondary education on the economic status of persons who are deaf or hard of hearing. Journal of deaf studies and deaf education. 2011;16(4):524-36.

3. Lazard D, Lee H, Gaebler M, Kell C, Truy E, Giraud A-L. Phonological processing in post-lingual deafness and cochlear implant outcome. Neuroimage. 2010;49(4):344351.

4. Mehra S, Eavey RD, Keamy DG. The epidemiology of hearing impairment in the United States: newborns, children, and adolescents. Otolaryngology-Head and Neck Surgery. 2009;140(4):461-72.

5. Bubbico L, Rosano A, Spagnolo A. Prevalence of prelingual deafness in Italy. Acta otorhinolaryngologica italica. 2007;27(1):17.

6. Mlynski R, Plontke S. Cochlear implants in children and adolescents. 2013;61(5):388-98.

7. Moon IS, Park S, Kim H-N, Lee W-S, Kim SH, Kim J-H, et al. Is there a deafness duration limit for cochlear implants in postlingual deaf adults, Acta oto-laryngologica. 2014;134(2):17380.

8. Kobosko J, Jedrzejczak WW, Pilka E, Pankowska A, Skarzynski H. Satisfaction With Cochlear Implants in Postlingually Deaf Adults and Its Nonaudiological Predictors: Psychological Distress, Coping Strategies, and Self-Esteem. Ear and hearing. 2015;36(5):605-18. 
9. Lassaletta L, Castro A, Bastarrica M, de Sarria MJ, Gavilán J. Quality of life in postlingually deaf patients following cochlear implantation. European Archives of Oto-Rhino-Laryngology and Head \& Neck. 2006;263(3):267-70.

10. Hoffman HJ, Dobie RA, Losonczy KG, Themann CL, Flamme GA. Declining Prevalence of Hearing Loss in US Adults Aged 20 to 69 Years. JAMA Otolaryngology-Head \& Neck Surgery. 2016.

11. Hajloo N, Ansari S. Prevalence and causes of hearing handicap in Ardabil province, Western Iran. Bimonthly Audiology-Tehran University of Medical Sciences. 2011;20(1):116-27.

12. Akhavanfar S. DA. Comparison of auditory performance and speech production skills after cochlear implantation in deaf children with \& without inner ear anomalies Audiology journal. 2004;18(3):427-38.

13. Ringdahl A, Grimby A. Severe-profound hearing impairment and health-related quality of life among post-lingual deafened Swedish adults. Scandinavian audiology. 2000;29(4):26675.

14. Angeli SI, Yan D, Telischi F, Balkany TJ, Ouyang XM, Du $\mathrm{LL}$, et al. Etiologic diagnosis of sensorineural hearing loss in adults. Otolaryngology-Head and Neck Surgery. 2005;132(6):890-5

15. Ahmad FI, DeMason CE, Teagle HF, Henderson L, Adunka OF, Buchman CA. Cochlear implantation in children with postlingual hearing loss. The Laryngoscope. 2012;122(8):1852-7.

16. Tanamati LF, Bevilacqua MC, Costa OA. Cochlear implant in postlingual children: functional results 10 years after the surgery. Brazilian journal of otorhinolaryngology. 2012;78(2):103-10.
17. Manrique M, Espinosa J, Huarte A, Molina M, Garcia-Tapia $\mathrm{R}$, Artieda J. [Cochlear implants in post-lingual persons: results during the first five years of the clinical course]. Acta otorrinolaringologica espanola. 1997;49(1):19-24.

18. Hamzavi J, Baumgartner W-d, Pok SM, Franz P, Gstoettner W. Variables affecting speech perception in postlingually deaf adults following cochlear implantation. Acta otolaryngologica. 2003;123(4):493-8.

19. Kraaijenga V, Smit A, Stegeman I, Smilde J, van Zanten G, Grolman W. Factors that influence outcomes in cochlear implantation in adults, based on patientrelated characteristics-a retrospective study. Clinical Otolaryngology. 2016.

20. Cao K, Wei C, Jin X, Chen X, Wang Y. Clinical review of cochlear implant in 533 cases. Zhonghua er bi yan hou ke za zhi. 2004;39(10):579-83.

21. Damen GW, Beynon AJ, Krabbe PF, Mulder JJ, Mylanus EA. Cochlear implantation and quality of life in postlingually deaf adults: long-term follow-up. Otolaryngology-Head and Neck Surgery. 2007;136(4):597-604.

22. Bittencourt AG, Ikari LS, Torre AAGD, Bento RF, Tsuji RK, Brito Neto RVd. Post-lingual deafness: benefits of cochlear implants vs. conventional hearing aids. Brazilian journal of otorhinolaryngology. 2012;78(2):124-7.

23. de Brito R, Bittencourt AG, Goffi-Gomez MV, Magalhães AT, Samuel P, Tsuji RK, et al. Cochlear implants and bacterial meningitis: A speech recognition study in paired samples. International archives of otorhinolaryngology. 2013;17(01):057-61.

24. Portmann D, Felix F, Negrevergne M, Bourdin M, Lagourgue $P$, Coulomb-Faye $F$, et al. Bilateral cochlear implantation in a patient with long-term deafness. Revue de laryngologieotologie-rhinologie. 2006;128(1-2):65-8. 